\title{
Ethylazinphos Interaction with Membrane Lipid Organization Induces Increase of Proton Permeability and Impairment of Mitochondrial Bioenergetic Functions
}

\author{
Romeu A. Videira, ${ }^{*} \dagger$ Maria C. Antunes-Madeira,* and Vítor M. C. Madeira*,1 \\ *Centro de Neurociências e Biologia Celular, Departamento de Zoologia, Universidade de Coimbra, 3004-517 Coimbra, Portugal; and \\ $\dagger$ Departamento de Ambiente, Escola Superior de Tecnologia, Instituto Superior Politécnico de Viseu, 3500 Viseu, Portugal
}

Received April 4, 2001; accepted June 12, 2001

Ethylazinphos Interaction with Membrane Lipid Organization Induces Increase of Proton Permeability and Impairment of Mitochondrial Bioenergetic Functions. Videira, R. A ., Antunes-Madeira, M. C., and Madeira, V. M. C. (2001). Toxicol. Appl. Pharmacol. 175, 209-216.

Ethylazinphos increases the passive proton permeability of lipid bilayers reconstituted with dipalmitoylphosphatidylcholine (DPPC) and mitochondrial lipids. A sharp increase of proton permeability is detected at insecticide/lipid molar ratios identical to those inducing phase separation in the plane of DPPC bilayers, as revealed by differential scanning calorimetry (DSC). E thylazinphos progressively depresses the transmembrane potential $(\Delta \Psi)$ of mitochondria supported by piruvate/malate, succinate, or ascorbate/T MPD. Additionally, a decreased depolarization induced by ADP depends on ethylazinphos concentration, reflecting a phosphorylation depression. This loss of phosphorylation is a consequence of a decreased $\Delta \Psi$. A decreased respiratory control ratio is also observed, since ethylazinphos stimulates state 4 respiration and inhibits ADPstimulated respiration (state 3). E thylazinphos concentrations up to $100 \mathrm{nmol} / \mathrm{mg}$ mitochondrial protein increase the rate of state 4 together with a decrease in $\Delta \Psi$, without significant perturbation of state 3 and carbonyl cyanide-p-trifluoromethoxyphenylhydrazone (FCCP)-uncoupled respiration. For increased insecticide concentrations, the state 3 and FCCP-uncoupled respiration are inhibited to approximately the same extent. The perturbations are more pronounced when the energization is supported by pyruvate/ malate and less effective when succinate is used as substrate. The present data, in association with previous DSC studies, indicate that ethylazinphos, at concentrations up to $100 \mathrm{nmol} / \mathrm{mg}$ mitochondrial protein, interacts with the lipid bilayer of mitochondrial membrane, changing the lipid organization and increasing the proton permeability of the inner membrane. The increased proton permeability explains the decreased oxidative phosphorylation coupling. Resulting disturbed ATP synthesis may significantly underlie the mechanisms of ethylazinphos toxicity, since most of cell energy in eukaryotes is provided by mitochondria. ๑ 2001 Academic Press

To whom correspondence should be addressed. Fax : +351-239826798; E-mail: vmc@ci.uc.pt.
Key Words: ethylazinphos; membrane organization; proton permeability; mitochondrial respiration; transmembrane potential; oxidative phosphorylation.

The two major components of the biological membranes are lipids and proteins. Together they determine the organization and function of membranes. These dynamic and versatile barriers are, in turn, involved in most cellular functions (Kinnunen, 1991). A large number of drugs with different chemical structures and pharmacological effects are able to accumulate in membrane lipid domains, changing their organization and physical properties and, consequently, their function (Sikkema et al., 1995; Mouritsen and Jørgensen, 1998), since the preservation of an exact degree of organization is essential for most membrane activities (Eze, 1990; Mouritsen and Jørgensen, 1998; Williams, 1998). Examples of the above compounds include anesthetics, calcium channel blocking drugs, alcohols, and insecticides (Makriyannis et al., 1986; Bae et al., 1989; Barry and Gawrisch, 1994; Mavromoustakos et al., 1995; Antunes-Madeira et al., 1996). In particular, the influence of organochlorine and organophosphorus insecticides on the physical properties of lipid bilayers and implications at the level of membrane cell function have been studied in our laboratory (Antunes-Madeira and Madeira, 1993; AntunesMadeira et al., 1994, 1996; Videira et al., 1996, 1999a,b; Lopes et al., 1997; Donato et al., 1997).

It is largely accepted that the basic mechanism of acute toxicity of organophosphorus compounds is associated with the specific inhibition of acetylcholinesterase (Ohkawa, 1982). However, the current understanding of how these compounds interact with the acetylcholinesterase is incomplete (Kardos and Sultatos, 2000), since the capacity to phosphorylate the enzyme appears to decrease as the organophosphorus concentration increases. On the other hand, the molecular mechanisms of the persistent signs underlying chronic intoxication induced by these compounds have not been elucidated. The toxicity at the cellular level has been demonstrated in several immortal cell lines (Veronesi and Ehrich, 1993; Swann et al., 1996). 
Alterations at subcellular level have also been detected, namely, inhibition of synaptosomal ATPases (Brown and Sharma, 1976), perturbation of membrane erythrocyte permeability (Antunes-Madeira et al., 1981), perturbation of $\mathrm{Ca}^{2+}-$ pump activity of sarcoplasmic reticulum (Antunes-Madeira and Madeira, 1982), and perturbation of mitochondrial functions (Sitkiewicz et al., 1980; Moreno and Madeira, 1990). These perturbations are detected for insecticide concentrations that did not inhibit acetylcholinesterase (Sitkiewicz et al., 1980). Furthermore, it has been demonstrated that the subcellular events that initiate the citotoxicity in processes like apoptosis and necrosis are connected with structural and functional alterations in mitochondrial membranes (Susin et al., 1998; Pedersen, 1999). Therefore, these membranes are important putative target sites for organophosphorus insecticides, since they accumulate preferentially into cholesterol-poor membranes, e.g., mitochondria (Videira et al., 1996; Antunes-Madeira and Madeira, 1989). A crucial mechanism for cell life is the mitochondrial oxidative phosphorylation coupled to an electrochemical gradient of $\mathrm{H}^{+}$(or $\mathrm{OH}^{-}$) across the inner membrane. Therefore, the mitochondrial activity and, consequently, cell life are seriously compromised by the perturbation of proton permeability. It has been demonstrated that changes in lipid packing and mobility affect membrane proton permeability (Zhang et al., 2000). Ethylazinphos (O,O-diethyl-S-[4oxo-1,2,3-benzotriazin-3-(4H)-ylmethyl]-phosphorodithioate), an organophosphorus insecticide, is expected to affect the proton permeability, since it alters the membrane lipid phase structure and organization (Videira et al., 1996, 1999a). Therefore, in the sequence of this previous work, the present study reports the effects of ethylazinphos on the proton permeability of lipid membranes and putative implications on basic mitochondrial functions that affect cell bioenergetics.

\section{MATERIALS AND METHODS}

Materials. Ethylazinphos was obtained from Supelco, Inc. (Bellefonte, PA). 5,6-Carboxyfluorescein (5,6-CF) was purchased from Molecular Probes Inc. (Eugene, OR). Dipalmitoylphosphatidylcholine (DPPC) and all the other chemicals were obtained from Sigma Chemical Co. (St. Louis, MO). All the compounds were of the highest commercially available quality.

Preparation of membranes for proton permeability measurements. Multilamellar vesicles of DPPC and of mitochondrial lipid extracts were prepared as described elsewhere (Antunes-Madeira and Madeira, 1993). Briefly, phospholipids were dissolved in $\mathrm{CHCl}_{3}$ in round-bottom flasks and the solvent was evaporated to dryness. The resulting dry thin lipid film on the wall of the round-bottom flask was hydrated with an appropriate volume of $50 \mathrm{mM} \mathrm{KCl}$, $5 \mathrm{mM}$ Tris-maleate, and $20 \mu \mathrm{M} 5,6-\mathrm{CF}, \mathrm{pH} 7.4$, and dispersed under $\mathrm{N}_{2}$ atmosphere by handshaking in a water bath set $7-10^{\circ} \mathrm{C}$ above the transition temperature for DPPC or at room temperature for mitochondrial lipids, and multilamellar vesicles were obtained. Large unilamellar vesicles (LUV) were prepared from multilamellar vesicles by standard extrusion techniques (Mayer et al., 1986). Thirteen repeated extrusions were performed through two stacked polycarbonated membranes of $200-\mathrm{nm}$ pore size. After extrusion, unencapsulated 5,6-CF was removed by passing the liposome suspension through a $1.4 \times$ 28-cm Sephadex G-50 column equilibrated with $50 \mathrm{mM} \mathrm{KCl}$ and $5 \mathrm{mM}$ Tris-maleate buffer, $\mathrm{pH}$ 7.4. The column was maintained at $23^{\circ} \mathrm{C}$ and the flow rate was $0.8 \mathrm{ml} / \mathrm{min}$. The fraction containing DPPC (LUV) with entrapped $5,6-\mathrm{CF}$ was collected. The diameters and the size distributions of vesicles were determined by dynamic light scattering using a Coulter Model N4 MD. The autocorrelation function was analyzed using Coulter software, applying the cumulant method and multiexponential fit method. The average diameter of the vesicles varied between 180 and $230 \mathrm{~nm}$. Phospholipid concentration in the final vesicle suspension was determined by measuring the inorganic phosphate (Bartlett, 1959) released after hydrolysis of dried lipids, at $180^{\circ} \mathrm{C}$, in $70 \%$ $\mathrm{HClO}_{4}$ (Böttcher et al., 1961).

Proton permeability. The proton influx into the lipid vesicles, due to the action of ethylazinphos, is inferred from the rate of 5,6-CF fluorescence decrease, which is a measure of the rate of $\mathrm{pH}$ decrease inside the vesicles (Szoka et al., 1979; Zeng et al., 1993). As the $\mathrm{pH}$ is lowered, 5,6-CF is protonated and its fluorescence intensity decreases.

Ethylazinphos was added in aliquots (up to $8 \mu \mathrm{l}$ ) from concentrated ethanolic solutions ( $50 \mathrm{mM}$ ) to $2 \mathrm{ml}$ of 5,6-CF-entrapped vesicle suspension, to give the insecticide/lipid molar ratios indicated in the figures. The mixture was incubated at the desired temperature for $10 \mathrm{~min}$ before the establishment of a $\mathrm{pH}$ gradient across the bilayer. Then, a $\mathrm{pH}$ gradient from 7.4 (inside) to 6.4 (outside) was established by adding aliquots of $0.1 \mathrm{M} \mathrm{HCl}$. The fluorescence intensity of 5,6-CF was monitored as a function of time in a Perkin-Elmer MPF-66 fluorescence spectrophotometer equipped with a thermostated cell holder. The fluorescence intensity of 5,6-CF was measured at $525 \mathrm{~nm}$ with an excitation at $489 \mathrm{~nm}$. The bandpass was $3 \mathrm{~nm}$ for excitation and emission beams (Zeng et al., 1993). The lipid concentration was nominally $0.2 \mathrm{mM}$ in all the samples examined. Furthermore, the experiments were carried out in the presence of valinomycin ( $1 \mu \mathrm{g} / \mathrm{mg}$ lipid) and potassium ions to prevent the formation of a membrane potential due to the electrogenic flux of protons into liposomes (Barchfeld and Deamer, 1985). At the concentration used (68.5 $\mathrm{mM}$ ), ethanol had no detectable effect on the proton permeability of lipid bilayers, which is in agreement with results described by others (Zeng et al., 1993).

The fluorescence intensity changes of 5,6-CF were fit to a double-exponential decay function using the error minimization procedure of the KaleidaGraph software with a $\chi^{2}$ between successive iterations less than $0.001 \%$. The curve-fit equation used was

$$
F(t)=A_{1} \exp \left(-k_{1} t\right)+A_{2} \exp \left(-k_{2} t\right)
$$

where $A_{1}$ and $A_{2}$ are the preexponential factors and $k_{1}$ and $k_{2}$ are the rate constants. Calculating the kinetic global constant as the average rate constant $k=A_{1} k_{1}+A_{2} k_{2}$ (Zeng et al. 1993) and the vesicle radii determined by light-scattering methodology, the proton permeability $(P)$ can be calculated from the equation (Elamrani and Blume, 1983)

$$
P=k V / S=k R / 3
$$

where $R, V$, and $S$ are the radius, volume, and surface area of the vesicles, respectively.

Statistics. Significance was calculated using Student's $t$ test of paired comparisons.

Isolation of rat liver mitochondria. Sprague-Dawley rats, of either sex, weighting 200-300 $\mathrm{g}$ were used for the isolation of liver mitochondria following established procedures (Gazotti et al., 1979), with minor modifications. Homogenization medium contained $0.25 \mathrm{M}$ sucrose; $5 \mathrm{mM}$ Hepes, $\mathrm{pH}$ 7.4; 0.2 mM EGTA; $0.1 \mathrm{mM}$ EDTA; and $0.1 \%$ fatty acid-free bovine serum albumin (BSA). EDTA, EGTA, and BSA were omitted from the final washing medium, which was adjusted to $\mathrm{pH}$ 7.2. The final concentration of the mitochondrial protein was determined by the biuret method (Gornall et al., 1949) using BSA as standard.

Mitochondrial respiratory activity. Oxygen uptake by the isolated mitochondria was measured polarographically using a Clark oxygen electrode (Estabrook, 1967) connected to a suitable recorder, in a 1-ml thermostated 
water-jacketed closed chamber with magnetic stirring, at $25^{\circ} \mathrm{C}$. The standard respiratory medium consisted of $130 \mathrm{mM}$ sucrose, $50 \mathrm{mM} \mathrm{KCl}, 5 \mathrm{mM} \mathrm{MgCl}_{2}$, $5 \mathrm{mM} \mathrm{KH}_{2} \mathrm{PO}_{4}$, and $5 \mathrm{mM}$ Hepes, $\mathrm{pH}$ 7.2. Ethylazinphos was added in aliquots (a few microliters) from a concentrated ethanolic solution to $1 \mathrm{ml}$ of standard respiratory medium supplemented with mitochondria (1 $\mathrm{mg}$ protein) and allowed to incubate for $10 \mathrm{~min}$, before the addition of respiratory substrates, i.e., before the beginning of the respiratory activity. At this elapsed time, the effects of ethylazinphos are maximal. The respiratory control ratio (RCR) was calculated according to Chance and Williams (1956).

Membrane potential. A tetraphenylphosphonium $\left(\mathrm{TPP}^{+}\right)$-sensitive electrode, prepared according to Kamo et al. (1979), was used to estimate the potential across the mitochondrial membrane. $\mathrm{TPP}^{+}$uptake has been measured from the decreased $\mathrm{TPP}^{+}$concentration in the medium sensed by the electrode. The potential difference between the selective electrode and the reference (calomel electrode) was measured with an electrometer and recorded continuously in a Linear 1200 recorder. The voltage response of the $\mathrm{TPP}^{+}$electrode to $\log \left[\mathrm{TPP}^{+}\right]$was linear with a slope of $59 \pm 1$, at $25^{\circ} \mathrm{C}$, in good agreement with the Nernst equation.

Mitochondria (1 mg protein) were resuspended in $1 \mathrm{ml}$ of the standard respiratory medium (the same medium described for oxygen consumption experiments), at $25^{\circ} \mathrm{C}$, with constant stirring. $\mathrm{TPP}^{+}$was added to a final concentration of $3 \mu \mathrm{M}$, i.e., low enough to avoid any detectable toxic effects on mitochondria (Jensen and Gunter, 1984). Before the energization of mitochondria with the respiratory substrates, ethylazinphos (a few microliters) was added from a concentrated ethanolic solution and the suspension was incubated for $10 \mathrm{~min}$. The $\mathrm{TPP}^{+}$uptake was initiated by the addition of substrate (final concentration, $5 \mathrm{mM}$ ). The $\Delta \Psi$ was estimated as indicated by Kamo et al. (1979) from the equation

$$
\Delta \Psi(\mathrm{mV})=59 \log (v / V)-59 \log \left(10^{\Delta E / 59}-1\right)
$$

where $v, V$, and $\Delta E$ are the volumes of mitochondrial and incubation medium and the deflection of the electrode potential from the baseline, respectively. The matrix volume of $1.1 \mu \mathrm{l} / \mathrm{mg}$ protein was considered. The above equation was derived assuming that $\mathrm{TPP}^{+}$distribution between the mitochondria and the medium follows the Nernst equation and that the law of mass conservation is applicable. No correction was made for the passive binding of $\mathrm{TPP}^{+}$to the mitochondrial membranes, since the purpose of the experiments was to show relative changes in potentials rather than absolute values. Calibration runs in the presence of ethylazinphos excluded any direct interference of the insecticide on the electrode signal.

\section{RESULTS}

\section{Effects of Ethylazinphos on Proton Permeability in Large Unilamellar Vesicles}

Figure 1 represents the effects of increasing ethylazinphos concentrations on the normalized fluorescence intensity of 5,6-CF entrapped in DPPC (LUV), as a function of time. The fluorescence intensity of 5,6-CF decreases with time after a pH gradient from 6.4 (outside) to 7.4 (inside) is established. The decay of fluorescence intensity results from the decrease of $\mathrm{pH}$ inside the vesicles that reflects proton influx driven by the $\mathrm{pH}$ gradient (Szoka et al., 1979; Zeng et al., 1993). The beginning intensity of this decay is normalized to unit (normalized $F_{525} \mathrm{~nm}$ $=1$ ) and corresponds to the moment of the $\mathrm{pH}$ gradient establishment. The decrease in normalized fluorescence intensity for ethylazinphos/lipid molar ratios indicated in the figure follows a double-exponential decay as described under Materials and Methods. The double-exponential decay satisfactorily

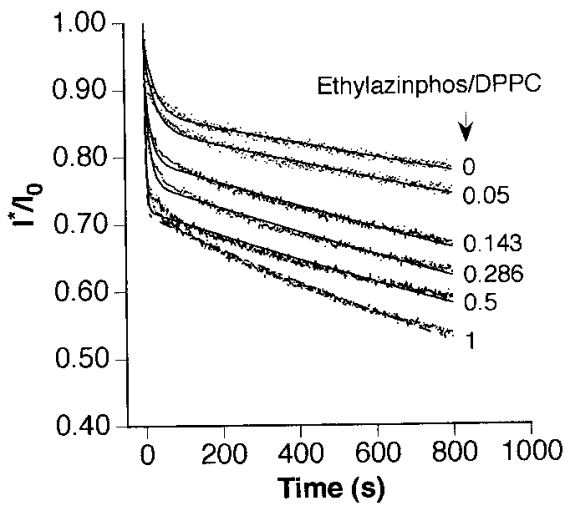

FIG . 1. Effects of increasing ethylazinphos concentrations on the normalized fluorescence intensity of 5,6-CF entrapped in DPPC large unilamellar vesicles as a function of time. The insecticide was added in aliquots (up to 8 $\mu \mathrm{l})$ from concentrated ethanolic solutions $(50 \mathrm{mM})$ to $2 \mathrm{ml}$ of 5,6-CF-entrapped vesicle suspension and the mixture was incubated for $10 \mathrm{~min}$, at $48^{\circ} \mathrm{C}$, before the establishment of a $\mathrm{pH}$ gradient, from 6.4 (outside) to 7.4 (inside), by the addition of aliquots of $0.1 \mathrm{M} \mathrm{HCl}$. The lipid concentration was nominally $0.2 \mathrm{mM}$ for all samples examined. Valinomycin ( $1 \mu \mathrm{g} / \mathrm{mg}$ lipid) was added to prevent the formation of a membrane potential due to the electrogenic flux of protons into the liposomes. The decrease in normalized fluorescence intensity reflects the influx of protons, which causes $\mathrm{pH}$ decrease inside the vesicles. As the ethylazinphos/lipid molar ratio increases from 0 to 1 , the fluorescence intensity decreases, which reflects an increase in proton influx.

fits the experimental data as reflected by low $\chi^{2}$ and high correlation factor $\left(R^{2}\right)$ (Table 1).

Figure 2 shows the effects of increasing ethylazinphos/lipid molar ratios on the global kinetic constant $(k)$ of the proton permeability of DPPC (LUV) solid phase at $30^{\circ} \mathrm{C}$ (filled circles), DPPC (LUV) fluid phase at $48^{\circ} \mathrm{C}$ (filled squares), and LUV reconstituted with mitochondrial lipids, at $37^{\circ} \mathrm{C}$ (open triangles). The global kinetic constant of proton permeability is proportional to the proton permeability $(P)$, since the volume and surface area of the vesicles are constant, because the insecticide does not change the vesicle size. In DPPC (LUV) solid and fluid phases, the proton permeability initially increases monotonically, with increasing ethylazinphos/lipid molar ratio up to 0.1 , followed by a sharp increase at insecticide/ lipid molar ratio of 0.143 . Above this value, the proton permeability increases again monotonically with ethylazinphos/lipid molar ratio. The critical ethylazinphos/lipid molar ratio $(0.143)$ is close to the ratio that induces phase separation in the plane of DPPC membranes, as previously reported (Videira et al., 1999a). The induced phase separation may thus contribute to the enhancement of the proton permeability across DPPC membranes. Additionally, the proton permeability determined in mitochondrial lipid dispersions shows a behavior identical to that observed in DPPC, with a critical point at insecticide/lipid molar ratio of 0.286 (Fig. 2). The results indicate that ethylazinphos also modifies lipid organization in mitochondrial lipid membranes, which may underlie the increase in proton permeability.

From Table 1, it is also clear that the permeability constant 
TABLE 1

F luorescence Intensity of 5,6-C F E ntrapped in D PPC

\begin{tabular}{lcccrr} 
Ethylazinphos/lipid & $A_{1}$ & $K_{1}$ & $A_{2}$ & $K_{2}$ & $R^{2}$ \\
\hline 0 & 0.1466 & 0.0403 & 0.8408 & 0.00014 & 0.984 \\
0.05 & 0.1572 & 0.0443 & 0.8270 & 0.00016 & 0.0145 \\
0.143 & 0.1700 & 0.0625 & 0.8150 & 0.00022 & 0.0166 \\
0.286 & 0.2228 & 0.1087 & 0.7660 & 0.00024 & 0.981 \\
0.5 & 0.2545 & 0.1541 & 0.7290 & 0.00026 & 0.0227 \\
1 & 0.2688 & 0.2706 & 0.7160 & 0.00037 & 0.0371 \\
\hline
\end{tabular}

Note. Data analysis, by double-exponential decay of fluorescence intensity of 5,6-CF entrapped in DPPC LUV upon the establishment of a pH gradient from 7.4 (inside) to 6.4 (outside), at $48^{\circ} \mathrm{C}$, for several ethylazinphos/lipid molar ratios.

for the fast component is significantly accelerated above molar ratios of 0.143 and that the constant for the slow component increases monotonically with the insecticide concentration. Therefore, the fast component determines to a large extent the behavior of the global kinetic constant. Although the first fast component is obviously important in the proton permeability, the second slow long-term component is probably more significant in terms of biochemical regulation.

Since the proton conductance of inner mitochondrial membrane modulates the coupling efficiency of oxidative phosphorylation (Rigoulet et al., 1998), the effects of ethylazinphos on the passive permeability to protons are likely to affect bioenergetic functions of mitochondria. Therefore, a correlation between ethylazinphos proton permeability perturbations and mitochondrial bioenergetic dysfunction has been searched in the following section.

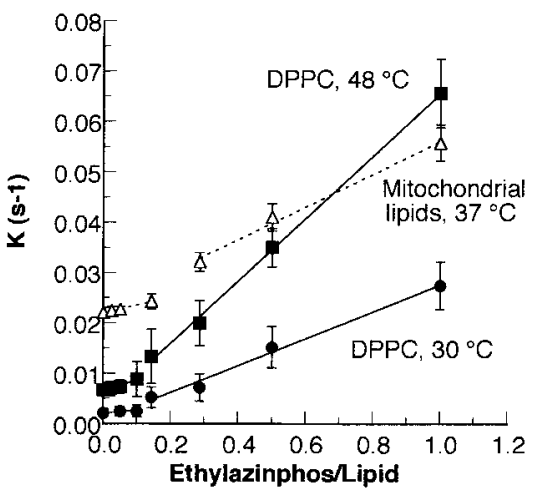

FIG. 2. Effects of increasing ethylazinphos/lipid molar ratios on the proton permeability, evaluated by the global kinetic constant $(K)$ of DPPC (LUV) solid phase, at $30^{\circ} \mathrm{C}$ (filled circles); DPPC (LUV) fluid phase, at $48^{\circ} \mathrm{C}$ (filled squares); and LUV reconstituted with mitochondrial lipid extracts, at $37^{\circ} \mathrm{C}$ (open triangles). A sharp increase in proton permeability is detected at ethylazinphos/lipid molar ratios of 0.143 and 0.286 for DPPC (LUV) and for mitochondrial LUV, respectively. Before and after these critical points the increase in permeability is monotonically. Each point represents the mean \pm SD from three to six independent experiments. Error bars for some data points are not represented, since they are encompassed by the size of the symbols. Permeability values for control samples were compared with those treated with ethylazinphos by Student's $t$ test. All the paired comparisons show significant differences $(p<0.0001)$.

\section{Interactions of Ethylazinphos with Liver Mitochondrial Bioenergetics}

Mitochondria develop a normal transmembrane potential $(\Delta \Psi)$ of $210-220 \mathrm{mV}$ (negative inside) upon addition of a respiratory substrate (Fig. 3). Addition of ADP causes a depolarization of $\Delta \Psi$ to about $-188 \mathrm{mV}$, since ATP-synthase uses $\Delta \Psi$ to phosphorylate ADP. After a short lag phase, when ADP phosphorylation takes place, the transmembrane potential repolarizes to the initial value (Fig. 3). The presence of ethylazinphos in the reaction medium depresses the $\Delta \Psi$ promoted by external substrates compared to control. Furthermore, the insecticide decreases the depolarization amplitude induced by ADP, lengthens the lag phase preceding repolarization, and decreases the repolarization rate (Fig. 3). These observations indicate that the energization and phosphorylation capacities of mitochondria are depressed by ethylazinphos. However, the effects are more pronounced when the global energization occurs at the expense of the three oxidation complexes after

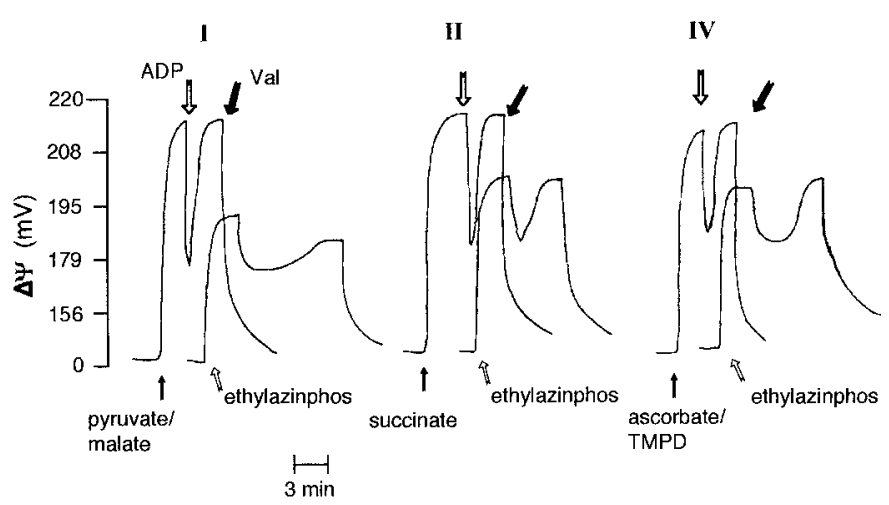

FIG . 3. Recordings of mitochondrial membrane potential $(\Delta \Psi)$ supported by three different substrates, in the absence and presence of ethylazinphos (200 $\mathrm{nmol} / \mathrm{mg}$ mitochondrial protein), at $25^{\circ} \mathrm{C}$. Mitochondria (1 mg protein) in $1 \mathrm{ml}$ of the standard respiratory medium supplemented with $3 \mu \mathrm{M} \mathrm{TPP}{ }^{+}$were energized with $5 \mathrm{mM}$ pyruvate/malate (activation complex I), $5 \mathrm{mM}$ succinate (activation complex II), or $5 \mathrm{mM}$ ascorbate/TMPD (activation complex IV). The mitochondrial suspensions were incubated for $10 \mathrm{~min}$ with ethylazinphos, before the energization. The phosphorylation was initiated with the addition of ADP $(100 \mu \mathrm{M})$. 

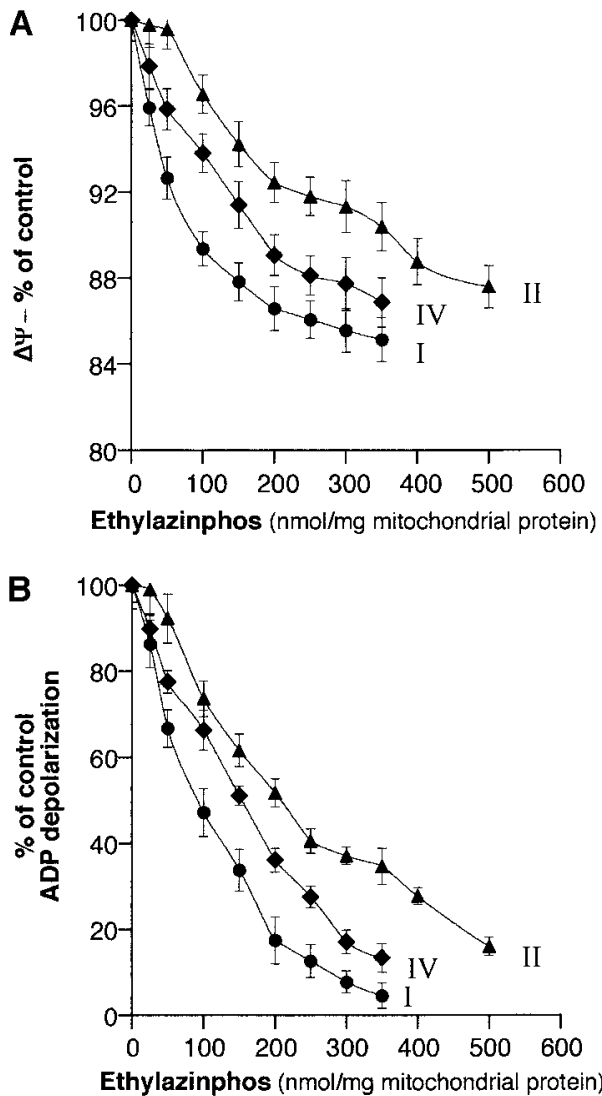

FIG. 4. Effects of increasing concentrations of ethylazinphos on the mitochondrial membrane potential $(\Delta \Psi)(\mathrm{A})$ and depolarization induced by ADP (B), supported by pyruvate/malate (I, circles), succinate (II, triangles), or ascorbate/TMPD (IV, diamonds). Relative decays were calculated assuming the $\Delta \Psi$ or the ADP depolarization in the absence of insecticide to be $100 \%$. Each point represents the mean \pm SD from three to five independent experiments. As revealed by Student's $t$ test for paired comparisons, significant differences $(p<0.0001)$ from the control have been achieved.

activation of complex I (pyruvate/malate). Smaller effects are observed when the energization is supported either by complexes II, III, and IV together (succinate activation) or by complex IV alone (activation by ascorbate/tetramethyl- $p$-phenylenediamine (TMPD)). In contrast to the energization supported by pyruvate/malate, the energization supported either by succinate or ascorbate/TMPD fully recovers, after ADP phosphorylation, to the level of state 4 .

Figure 4A shows that ethylazinphos progressively decreases the transmembrane potential of mitochondria supported by piruvate/malate, succinate, or ascorbate/TMPD, i.e., developed by activation of complexes I, II, and IV, respectively. As illustrated in Fig. 4, the effects are more pronounced when complex I is activated and less pronounced upon activation of complex II; intermediate effects are observed upon activation of complex IV. For instance, an ethylazinphos concentration of $100 \mathrm{nmol} / \mathrm{mg}$ mitochondrial protein depresses the membrane potential by $10.6,3.5$, and $6.2 \%$ with substrate oxidation at the level of complex I, II, and IV, respectively. Also, a decreased depolarization induced by ADP depends on ethylazinphos concentration (Fig. 4B), reflecting loss of mitochondrial phosphorylation efficiency. Comparing the results of Figs. 4A and 4B, it is concluded that the decay of the transmembrane potential parallels the decrease of depolarization following ADP addition. A detailed analysis of the results for complex I activation indicates that the decay of the $\Delta \Psi$ to $85 \%$ of the control results in a full collapse of the ADP depolarization, suggesting absence of ATP synthesis.

The concentration-dependent effects of ethylazinphos on oxygen consumption and RCR supported by the above three substrates are shown in Fig. 5. With all the substrates, ethylazinphos causes a dual effect: a net stimulation of state 4 respiration (filled squares) and inhibition of ADP-stimulated respiration, state 3 (filled triangles). Consequently, a marked drop of the respiratory control ratio is observed (Fig. 5, open circles). The respiratory basal rate (state 4$)$ supported by all the substrates increases significantly $(p<0.0001)$ up to $100 \mathrm{nmol}$ ethylazinphos/mg mitochondrial protein, especially upon activation of complex I. These ethylazinphos concentrations have almost no effect in the phosphorylating respiratory rate (state 3 ) and in the carbonyl cyanide- $p$-trifluoromethoxyphenylhydrazone (FCCP)-uncoupled respiration (Fig. 5). At concentrations above $100 \mathrm{nmol} / \mathrm{mg}$ mitochondrial protein, ethylazinphos depresses state 3 and FCCP-uncoupled respiration to approximately the same extent.

The effective concentrations used in the present study are difficult to correlate with the LD50 (RTECS, 1997) obtained with experimental mammals, since the distribution of ethylazinphos in tissues will depend on a variety of parameters, namely partition coefficients (Antunes-Madeira and Madeira, 1989; Videira et al. 1996). The phosphorothioate compounds in general, and ethylazinphos in particular, partition into cholesterol-poor membranes, such as mitochondria, to a greater extent than into cholesterol-rich brain microsomes, myelin, or erythrocytes. However, significant effects were observed in mitochondria bioenergetic for ethylazinphos concentrations of $100 \mathrm{nmol} / \mathrm{mg}$ mitochondrial protein, which are much lower than those needed to inhibit acetylcholinesterase by $50 \%$ (>800 nmol/mg microsomal protein) (Sitkiewicz et al., 1980; Davies and Holub, 1983). Therefore, the effects observed in mitochondria are likely to contribute to the toxicity of ethylazinphos.

\section{DISCUSSION}

Mitochondria support the energy-dependent regulation of several cell functions, namely intermediary metabolism, protein folding, ion regulation, cell motility, and cell proliferation (reviewed in Pedersen, 1999 and Wallace, 1999). Animal cells derive more than $90 \%$ of the energy from oxidative phosphorylation associated with the inner mitochondrial membrane (Mitchell, 1961). Thus, compounds that interact with mitochondrial membranes perturbing the coupling efficiency be- 

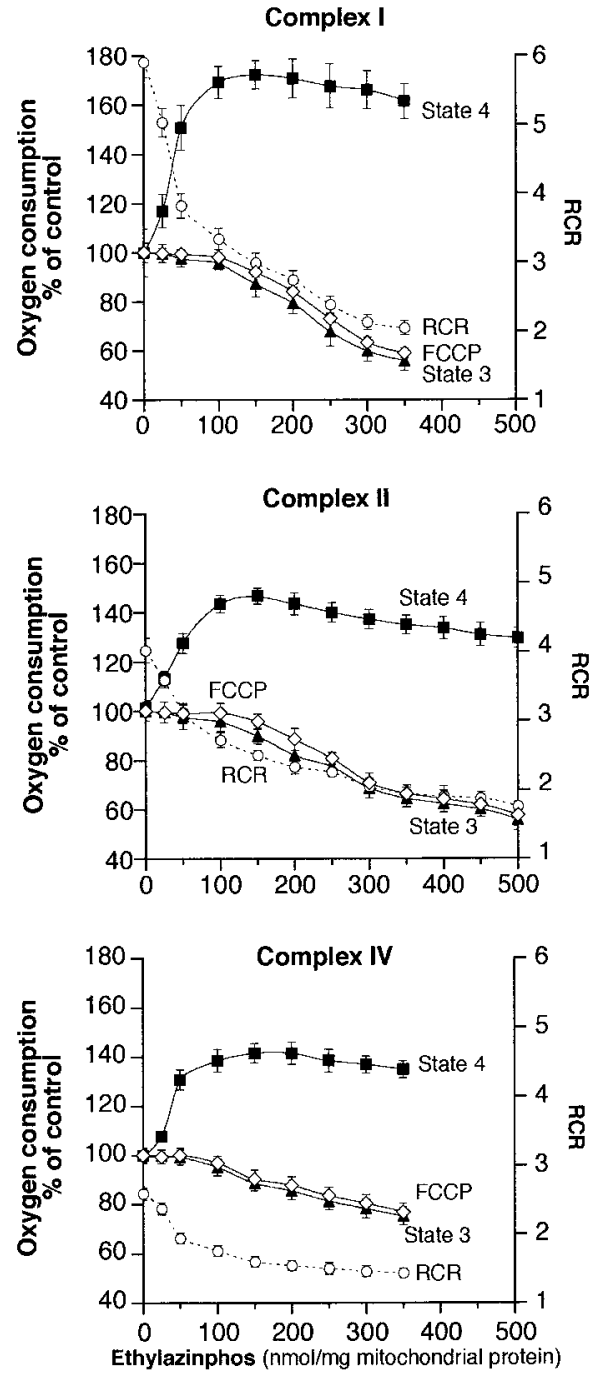

FIG. 5. Effects of increasing concentrations of ethylazinphos on the mitochondrial oxygen consumption supported by pyruvate/malate (activation complex I), succinate (activation complex II), or ascorbate/TMPD (activation complex IV). Mitochondria (1 $\mathrm{mg}$ protein) were incubated in $1 \mathrm{ml}$ of the respiratory standard medium. Ethylazinphos was added $10 \mathrm{~min}$ before starting state 4 respiration (filled squares). To induce state 3 respiration (filled triangles), ADP $(100 \mu \mathrm{M})$ was added. The FCCP-uncoupled respiration (open diamonds) was initiated by the addition of $1.5 \mu \mathrm{M}$ FCCP. Relative values were calculated assuming the oxygen consumption in the absence of insecticide to be $100 \%$. The respiratory control ratio (open circles and dotted lines) was calculated by the ratio of oxygen consumption in state 3 and state 4 . Each point represents the mean $\pm \mathrm{SD}$ from three to five independent experiments. As in Fig. 2 the SD for several data points are too small to be displayed by error bars.

tween oxidation and phosphorylation promote large bioenergetic deficits leading to the loss of several functions vital to the survival of the cell and the organism. Therefore, evaluation of ethylazinphos perturbations on mitochondrial structure and function are important for understanding the molecular mechanisms of its toxicity.

The present study demonstrates that ethylazinphos increases the proton permeability of DPPC and mitochondrial lipid bi- layers (Figs. 1 and 2). A substantial increase in proton permeability is detected for ethylazinphos concentrations that induce phase separation in the plane of DPPC bilayers (Videira et al., 1999a), as a consequence of the sharp increase of the constant for the fast component. Also Zeng et al. (1993) demonstrated that ethanol concentrations (1.2 to $1.8 \mathrm{M}$ ), which induce phase separation in the plane of the DPPC bilayers, concomitantly enhance the proton permeability. Therefore, the lateral organization of membrane lipids modulates the passive permeability to protons. On the other hand, these results indicate a specific contribution of the interfacial region between two phases in solute permeability. Indeed, it has been demonstrated that, in regions of two-phase coexistence, occurring at the main phase transition between gel and liquid-crystalline states, a sharp increase in solute permeability is observed (Block et al., 1976). Moreover, the transbilayer permeability can be used to predict membrane lipid heterogeneity (Xiang and Anderson, 1998) and its modification by xenobiotic compounds (Williams, 1998). Therefore, the break in proton permeability in mitochondrial lipid bilayers for a given ethylazinphos concentration (Fig. 2) may reflect a reorganization of membrane lipid domains, creating localized areas of altered permeability.

The ethylazinphos perturbations in mitochondrial functions were studied following the changes in membrane energization $(\Delta \Psi)$, supported by three different substrates, namely, pyruvate/malate, succinate, and ascorbate/TMPD, and in oxygen consumption. The effects on $\Delta \Psi$ are significant since it represents the main component of the electrochemical proton gradient, accounting for more than $90 \%$ of the total available respiratory energy (Nicholls, 1984).

Ethylazinphos progressively depresses $\Delta \Psi$ supported by all three substrates and decreases the depolarization amplitude induced by ADP. Consequently, the phosphorylation efficiency of mitochondria is depressed. These effects are more pronounced when the respiration is supported by pyruvate/malate compared with other substrates. Furthermore, the repolarization after ADP phosphorylation does not reach the characteristic state 4 level during pyruvate/malate oxidation. The total repolarization of the membrane upon the state 3 cycle, when succinate and ascorbate are oxidized, suggests that ethylazinphos does not disrupt ATP synthase itself. In turn, the lack of full repolarization with pyruvate/malate suggests that specific perturbations occur at the level of complex I or at the electron delivery from complex I to complex III, e.g., at quinone binding sites in complex I.

Ethylazinphos concentrations up to $100 \mathrm{nmol} / \mathrm{mg}$ mitochondrial protein increase the rate of state 4 without significant perturbations in state 3 respiration, an effect occurring simultaneously with a decrease in membrane potential. Therefore, for these concentrations, ethylazinphos increases the inner mitochondrial membrane permeability to protons without affecting the phosphorylation capacity in terms of the phosphorylation ratio $\mathrm{ADP} / \mathrm{O}$. However, for insecticide concentrations higher than $100 \mathrm{nmol} / \mathrm{mg}$ mitochondrial protein, the state 3 
respiration is inhibited. Thus, the inhibitory effect on phosphorylation efficiency in terms of energization $(\Delta \Psi)$ and RCR may result from uncoupling effects of ethylazinphos that are apparently linked to the increased permeability of mitochondrial membrane to protons, as inferred by the stimulation of state 4 respiration. This is supported by the increasing passive proton permeability induced by ethylazinphos in DPPC and mitochondrial lipid bilayers.

The depression of uncoupled respiration for ethylazinphos concentrations above $100 \mathrm{nmol} / \mathrm{mg}$ mitochondrial protein reflects the interaction of ethylazinphos with the mitochondrial redox complexes (Balaban, 1990). Moreover, state 3 respiration is depressed to approximately the same extent as uncoupled respiration, ruling out major interactions with the phosphorylation assembly (Tager et al., 1983). Therefore, the energetic perturbations, as evaluated by the RCR, are related to alterations induced by the insecticide in membrane lipid physicochemical and structural properties and also to direct effects on redox complexes, which may also depend on structural perturbations.

As discussed above, stimulation of state 4 by ethylazinphos up to $100 \mathrm{nmol} / \mathrm{mg}$ of mitochondrial protein reflects the increased proton permeability of the membrane. However, for higher concentrations, state 4 is slightly depressed as a consequence of inhibition of redox complexes.

Therefore, the inhibitory action of ethylazinphos on the phosphorylation efficiency of mitochondria is also related to perturbations in the electron delivery in and between redox complexes in addition to the increased permeability to protons. These data, concomitantly with the ability of ethylazinphos to induce phase separation in lipid membranes (Videira et al., 1999a), indicate that the resulting membrane heterogeneity favors the existence of discontinuous regions, not percolated (Almeida et al., 1992; Mukherjee and Maxfield, 2000) in a continuous membrane phase, making it difficult for the electron delivery.

The effects of ethylazinphos on proton permeability, membrane potential, and oxidative phosphorylation are a consequence of its ability to perturb the inner mitochondrial membrane structure and organization. The inhibitory action on mitochondrial oxidative phosphorylation may explain physiological changes (Gupta et al., 1994; Carlson and Ehrich, 1999) not related to inhibition of the cholinergic system. Therefore, the correlation between alterations in membrane organization and function provides novel insights to the understanding of the molecular mechanisms of ethylazinphos toxicity in particular and of insecticides in general.

\section{ACKN OW LEDGMENTS}

This work was supported by Grants Praxis 2/2.1/BIO/1156/94 and 2/2.1/ SAU/1400/95. Romeu A. Videira is the recipient of a Ph.D. Grant GGP XXI/BD/2834/96.

\section{REFERENCES}

Almeida, P. F. F., Vaz, W. L. C., and Thompson, T. E. (1992). Lateral diffusion and percolation in two-phase, two-component lipid bilayers: Topology of the solid-phase domains in-plane and across the lipid bilayer. Biochemistry 31, 7198-7210.

Antunes-Madeira, M. C., Carvalho, A. P., and Madeira, V. M. C. (1981). Interactions of insecticides with erythrocyte membranes. Pestic. Biochem. Physiol. 15, 79-89.

Antunes-Madeira, M. C., and Madeira, V. M. C. (1982). Interaction of insecticides with the $\mathrm{Ca}^{++}$-pump activity of sarcoplasmic reticulum. Pestic. Biochem. Physiol. 17, 185-190.

Antunes-Madeira, M. C., and Madeira, V. M. C. (1989). Membrane partitioning of organophosphorus and organochlorine insecticides and its implications for mechanisms of toxicity. Pestic. Sci. 26, 167-169.

Antunes-Madeira, M. C., and Madeira, V. M. C. (1993). Effects of DDE on the fluidity of model and native membranes: Implications for the mechanisms of toxicity. Biochim. Biophys. Acta 1149, 86-92.

Antunes-Madeira, M. C., Videira, R. A., and Madeira, V. M. C. (1994). Effects of parathion on membrane organization and its implications for the mechanisms of toxicity. Biochim. Biophys. Acta 1190, 149-154.

Antunes-Madeira, M. C., Videira, R. A., Lopes, V. I. C. F., and Madeira, V. M. C. (1996). Toxicity of organophosphorus insecticides: Alteration of membrane fluidity. Med. Sci. Res. 24, 753-756.

Bae, S-J., Kitamura, S., Herbette, L. J., Sturtevant, J. M. (1989). The effects of calcium channel blocking drugs on the thermotropic behavior of dimyristoylphosphatidyl-choline. Chem. Phys. Lipids 51, 1-7.

Balaban, R. S. (1990). Regulation of oxidative phosphorylation in the mammalian cell. Am. J. Physiol. 258, C377-C389.

Barchfeld, G. L., and Deamer, D. W. (1985). The effect of general anesthetics on the proton and potassium permeabilities of liposomes. Biochim. Biophys. Acta 819, 161-169.

Barry, J. A., and Gawrisch, K. (1994). Direct NMR evidence for ethanol binding to the lipid-water interface of phospholipid bilayers. Biochemistry 33, 8082-8088.

Bartlett, G. R. (1959). Phosphorus assay in column chromatography. J. Biol. Chem. 234, 466-468.

Block, M. C., Van Deenen, L. L. M., and DeGier, J. (1976). Effects of the gel to liquid crystalline phase transition and the osmotic behavior of phosphatidylcholine liposomes. Biochim. Biophys. Acta 433, 1-12.

Böttcher, C. J. F., Van Gent, C. M., and Pires, C. (1961). A rapid and sensitive sub-micro phosphorus determination. Anal. Chim. Acta 24, 203-204.

Brown, H. R., and Sharma, R. P. (1976). Synaptosomal adenosine triphosphatase (ATPase) inhibition by organophosphates. Experientia 32, 15401542 .

Carlson, K., and Ehrich, M. (1999). Organophosphorus compound-induced modification of SH-SY5Y human neuroblastoma mitochondrial transmembrane potential. Toxicol. Appl. Pharmacol. 160, 33-42.

Chance, B., and Williams, G. R. (1956). The respiratory chain and oxidative phosphorylation, Adv. Enzymol. 17, 65-134.

Davies, D. B., and Holub, B. J. (1983). Comparative effects of organophosphorus insecticides on the activities of acetylcholinesterase, diacylglycerol kinase and phosphatidylinositol phosphodiesterase in rat brain microsomes. Pestic. Biochem. Physiol. 20, 92-99.

Donato, M. M., Jurado, A. S., Antunes-Madeira, M. C., and Madeira, V. M. C. (1997). Bacillus stearothermophilus as a model to evaluate membrane toxicity of a lipophilic environmental pollutant (DDT). Arch. Environ. Contam. Toxicol. 33, 109-116.

Elamrani, K., and Blume, A. (1983). Effects of the lipid phase transition on the 
kinetics of $\mathrm{H}^{+} / \mathrm{OH}^{-}$diffusion across phosphatidic acid bilayers. Biochim. Biophys. Acta 727, 22-30.

Estabrook, R. W. (1967). Mitochondrial respiratory control and the polarographic measurements of ADP/O ratios. Methods Enzymol. 10, 41-47.

Eze, M. O. (1990). Consequences of the lipid bilayer to membrane-associated reactions. J. Chem. Educ. 67, 17-19.

Gazotti, P., Malmstrom, K., and Crompton, M. (1979). A laboratory manual on transport and bioenergetics. In Membrane Biochemistry (F. Carafoli and G. Semenza, Eds.), pp. 62-79. Springer-Verlag, New York.

Gornall, A. G., Bardawill, C., and David, M. M. (1949). Determination of serum proteins by means of the biuret reaction. J. Biol. Chem. 177, 751-756.

Gupta, R. C., Goad, J. T., and Kadel, W. L. (1994). Cholinergic and noncholinergic changes in skeletal muscles by carbofuran and methylparathion. $J$. Toxicol. Environ. Health 43, 291-304.

Jensen, B. D., and Gunter, T. E. (1984). The use of tetraphenylphosphonium $\left(\mathrm{TPP}^{+}\right)$to measure membrane potentials in mitochondria: Membrane binding and respiratory effects. Biophys. J. 45, 92a.

Kamo, N., Muratsugu, M., Hongoh, R., and Kobatake, V. (1979). Membrane potential of mitochondria measured with an electrode sensitive to tetraphenylphosphonium and relationship between proton electrochemical potential and phosphorylation potential in steady state. J. Membr. Biol. 49, 105-121.

Kardos, S. A., and Sultatos, L. G. (2000). Interactions of the organophosphorus paraoxon and methylparaoxon with mouse acetylcholinesterase. Toxicol. Sci. 58, 118-126.

Kinnunen, P. K. J. (1991). On the principles of functional ordering in biological membranes. Chem. Phys. Lipids 57, 375-399.

Lopes, V. I. C. F., Antunes-Madeira, M. C., and Madeira, V. M. C. (1997). Effects of methylparathion on membrane fluidity and its implications for the mechanisms of toxicity. Toxicol. in Vitro 11, 337-345.

Makriyannis, A., Siminovitch, D. J., Das Gupta, S. K., and Griffin, R. G. (1986). Studies on the interaction of anesthetic steroids with phosphatidylcholine using ${ }^{2} \mathrm{H}$ and ${ }^{13} \mathrm{C}$ solid state NMR. Biochim. Biophys. Acta 859, 49-55.

Mavromoustakos, T., Yang, D. P., and Makriyannis, A. (1995). Effects of anesthetic steroid alphaxalone and its inactive D16-analog on the thermotropic properties of membrane bilayers: A model for membrane perturbation. Biochim. Biophys. Acta 1239, 257-264.

Mayer, L. D., Hope, M. J., and Cullis, P. R. (1986). Vesicles of variable sizes produced by a rapid extrusion procedure. Biochim. Biophys. Acta 858, 161-168.

Mitchell, P. (1961). Coupling of phosphorylation to electron and hydrogen transfer by a chemi-osmotic type mechanism. Nature 191, 144-148.

Moreno, A. J. M., and Madeira, V. M. C. (1990). Interference of parathion with mitochondrial bioenergetics. Biochim. Biophys. Acta 1015, 166-174.

Mouritsen, O. G., and Jørgensen, K. (1998). A new look at lipid-membrane structure in relation to drug research. Pharm. Res. 15, 1507-1509.

Mukherjee, S., and Maxfield, F. R. (2000). Role of membrane organization and membrane domains in endocytic lipid trafficking. Traffic 1, 203-211.

Nicholls, D. G. (1984). Mechanisms of energy transduction. In Bioenergetics (L. Ernster, Ed.), pp. 29-48. Elsevier, Amsterdam/New York.

Ohkawa, H. (1982). Stereoselectivity of organophosphorus insecticides. In
Insecticide Mode of Action (J. R. Coats, Ed.), pp. 163-185. Academic Press, London.

Pedersen, P. L. (1999). Mitochondrial events in the life and death of animal cells: A brief overview. J. Bioenerg. Biomembr. 31, 291-304.

Rigoulet, M., Devin, A., Espié, P., Guérin, B., Fontain, E., Piquet, M. A., Nogueira, V., and Leverve, X. (1998). Flux-force relationships in intact cells: A helpful tool for understanding the mechanism of oxidative phosphorylation? Biochim. Biophys. Acta 1365, 117-124.

RTECS (1997). Registry of Toxic Effects of Chemical Substances. NIOSH, U.S. Department of Health and Human Services, Cincinnati, OH.

Sikkema, J., De Bont, J. A. M., and Poolman, B. (1995). Mechanisms of membrane toxicity of hydrocarbons. Microbiol. Rev. 59, 201-222.

Sitkiewicz, D., Skonieczna, M., Krzywicka, K. Dziedzic, E. Staniszewska, K., and Bicz, W. (1980). Effect of organophosphorus insecticides on the oxidative processes in rat brain synaptosomes. J. Neurochem. 34, 619-626.

Susin, S. A., Zamzami, N., and Kroemer, G. (1998). Mitochondria as regulators of apoptosis: Doubt no more. Biochim. Biophys. Acta 1366, 151-156.

Swann, J. M., Schultz, T. W., and Kennedy, J. R. (1996). The effects of organophosphorus insecticides dursban and lorsban on the ciliated epithelium of the frog palate in vitro. Arch. Environ. Contam. Toxicol. 30, $188-194$.

Szoka, F. C., Jacobson, K., and Papahadjopoulos, D. (1979). The use of aqueous space markers to determine the mechanism of interaction between phospholipid vesicles and cells. Biochim. Biophys. Acta 551, 295-303.

Tager, J. M., Wanders, R. J. A., Groen, A. K., Kunz, W., Bohnensack, R., Kuster, U., Letko, G., Duszynski, J., and Wojtczak, L. (1983). Control of mitochondrial respiration. FEBS Lett. 151, 1-9.

Veronesi, B., and Ehrich, M. (1993). Differential cytotoxic sensitivity in mouse and human cell lines exposed to organophosphate insecticides. Toxicol. Appl. Pharmacol. 120, 240-246.

Videira, R. A., Antunes-Madeira, M. C., and Madeira, V. M. C. (1996). Interaction of ethylazinphos with the physical organization of model and native membranes. Biochim. Biophys. Acta 128, 65-72.

Videira, R. A., Antunes-Madeira, M. C., and Madeira, V. M. C. (1999a). Biophysical perturbations induced by ethylazinphos in lipid membranes. Chem. Phys. Lipids 97, 139-153.

Videira, R. A., Antunes-Madeira, M. C., and Madeira, V. M. C. (1999b). Perturbations induced by $\alpha$ - and $\beta$-endosulfan in lipid membranes: A DSC and fluorescence polarization study. Biochim. Biophys. Acta 1419, 151-153.

Wallace, D. C. (1999). Mitochondrial diseases in man and mouse. Science 283, 1482-1488.

Williams, E. E. (1998). Membrane lipids: What membrane physical properties are conserved during physicochemically-induced membrane restructuring? Am. Zool. 38, 280-290.

Xiang, T. X., and Anderson, B. D. (1998). Phase structures of binary lipid bilayers as revealed by permeability of small molecules. Biochim. Biophys. Acta 1370, 64-76.

Zeng, J., Smith, K. E., and Chong, P. L. G. (1993). Effects of alcohol-induced lipid interdigitation on proton permeability in L- $\alpha$-dipalmitoylphosphatidylcholine vesicles. Biophys. J. 65, 1404-1414.

Zhang, G. J., Liw, H. W., Yang, Y. G., and Zheng, Y. Z. (2000). Influence of membrane physical state on the lysosomal proton permeability. J. Membr. Biol. 175 53-62. 